\title{
The preclinical discovery and development of opicapone for the treatment of Parkinson's disease
}

\author{
Miren Ettcheto $* 1,2,3,4$, Oriol Busquets ${ }^{1,2,3,4}$, Elena Sánchez-Lopez ${ }^{4,5,6}$, Amanda Cano ${ }^{4,5,6}$, \\ Patricia R. Manzine 1,3,4,7, Ester Verdaguer ${ }^{3,4,8}$, Jordi Olloquequi ${ }^{10}$, Carme Auladell ${ }^{3,4,8}$ Jaume \\ Folch ${ }^{2,3}$ and Antoni Camins $* 1,3,4,10$
}

\footnotetext{
${ }^{1}$ Departament of Pharmacology, Toxicology and Therapeutic Chemistry, Faculty of Pharmacy and Food Science, University of Barcelona, Barcelona, Spain.

${ }^{2}$ Department of Biochemistry and Biotechnology, Faculty of Medicine and Life Science, University Rovira i Virgili, Reus, Spain.

3 Biomedical Research Networking Centre in Neurodegenerative Diseases (CIBERNED), Madrid, Spain.

${ }^{4}$ Institute of Neuroscience, University of Barcelona, Barcelona, Spain.

${ }^{5}$ Institute of Nanoscience and Nanotechnology (IN2UB), University of Barcelona, Barcelona, Spain.

${ }^{6}$ Department of Pharmacy, Pharmaceutical Technology and Physical Chemistry, Faculty of Pharmacy and Food Science, University of Barcelona, Barcelona, Spain

${ }^{8}$ Department of Cellular Biology, Physiology and Immunology, Faculty of Biology, University of Barcelona, Barcelona, Spain.
}

${ }^{9}$ Department of Gerontology, Federal University of São Carlos (UFSCar), São Carlos 13565-905, Brazil.

${ }^{10}$ Laboratory of Cellular and Molecular Pathology, Instituto de Ciencias Biomédicas, Facultad de

Ciencias de la Salud, Universidad Autónoma de Chile, Talca, Chile.

*These two authors are senior coauthors and contributed equally to the manuscript.

Correspondence and reprint requests to Antoni Camins PhD, Unitat de Farmacologia i Farmacognòsia, Facultat de Farmàcia i Ciències de l'Alimentació, Universitat de Barcelona, Barcelona, Spain. Av. Joan XXIII 27/31, E-08028 Barcelona, Spain. Tel: +34 93 4024531, Fax: +34 934035982, Mail: camins@ub.edu 


\section{Highlights}

- Opicapone is a third generation COMT inhibitor.

- Opicapone is administered once a day.

- Opicapone has high affinity to COMT as well as a slow dissociation constant and a prolonged duration of action.

- Opicapone is a better therapeutic agent than both ENT and TOL in patients with PD experiencing end-of-dose-fluctuations when used in combination with L-DOPA/DDI.

- Opicapone reduces "off" time and increases "on" time.

- The therapeutic benefits of Opicapone 50mg are maintained without dose adjustment of L-DOPA/DDI for at least one year. 


\begin{abstract}
Introduction: Opicapone (OPC) is a well-established catechol-O-methyltransferase (COMT) inhibitor that is approved for the treatment of Parkinson's disease (PD) associated with L-DOPA / L-amino acid decarboxylase inhibitor (DDI) therapy allowing for prolonged activity due to a more continuous supply of L-DOPA in the brain. Thus, OPC decreases fluctuation in L-DOPA plasma levels and favours more constant central dopaminergic receptor stimulation, thus improving PD symptomatology.

Areas covered: This review evaluates the preclinical development, pharmacology, pharmacokinetics and safety profile of OPC. Data were extracted from published preclinical and clinical studies published on PUBMED and SCOPUS (Search period: 2000-2019). Clinical and post-marketing data were also evaluated.
\end{abstract}

Expert opinion: OPC is a third generation COMT inhibitor with a novel structure. It has an efficacy and tolerability superior to its predecessors, tolcapone (TOL) and entacapone (ENT). It also provides a safe and simplified drug regimen that allows neurologists to individually adjust the existing daily administration of L-DOPA. OPC is indicated as an adjunctive therapy to LDOPA/DDI in patients with PD and end-of-dose motor fluctuations who cannot be stabilised on those combinations.

\footnotetext{
Abbre viations: 3-OMD, 3-O-methyldopa; 6-OHDA, 6-hydroxydopamine; BG, basal ganglia; COMT, Catechol-O-methyltransferase; DDI, decarboxylase inhibitors; ENT, Entacapone; FDA, Food and Drug Administration; MPTP, 1-methyl-4-phenyl-1,2,3,6-tetrahydropyridine; OPC, Opicapone; PD, Parkinson's disease; TOL, Tolcapone; GDNF, Glial cell-line-derived neurotrophic factor; NTN, neurturin; ICV, Intracerebroventricular; PDUFA, Prescription Drug User Fees Act; EMA, European Medicine Administration; AE, Adverse event BG, Basalganglia. $\mathrm{QD}$, once a day.
} 


\section{Introduction}

Idiopathic Parkinson's disease (PD) is a progressive neurodegenerative disorder clinically characterized by the presence of loss of motor control, slowness of movement, tremors at rest and rigidity [1]. Age is the most important risk factor, affecting approximately $1 \%$ of the population over 60 years. In addition to causing loss of motor control, patients show non-motor symptoms such as mood disorders, constipation, cognitive impairment, sleep disorders and difficulties with speech and swallowing [1-4]. Therefore, PD has generally been considered a motor disorder. The clinical manifestations are subject to the severity of neuron loss in the striatum region, specifically in the substantianigra, resulting in neurophysiological changes in basal ganglia (BG) activity, which underlie the cardinal symptoms of the disease [3-5]. From a neurochemical perspective, there is a striatal dopamine decrease, together with neuronal loss, which is responsible for approximately $70 \%$ of the clinical manifestations of idiopathic PD.

Currently the pharmacological approach to PD treatment is exclusively symptomatic. Treatment also varies significantly depending on the stage of the disease and patient age. However, the common therapeutic aim is to improve the functional situation and of patients [5-10]. There are several medications utilized for PD [8-10].

Amongst the first-line drugs, L-DOPA, an endogenous dopamine precursor administered in association with peripheral decarboxylase inhibitors (DDI), is considered to be the most effective drug for motor symptoms [8-10].

However, the problem with L-DOPA is that, over time, a high percentage of patients develop what is known as "motor fluctuations, " an end-of-dose deterioration, or "wearing-off, " with or without dyskinesias) [7-9]. The frequency of the development of these conditions varies depending on the age of the patient, the duration of treatment and the dose of L-DOPA used [1]. Progression of the disease requires that the clinician gradually increase the dose of L-DOPA to achieve adequate motor control. This in turn leads to the development of motor fluctuations. Motor fluctuations demonstrate a temporal relationship with L-DOPA levels. Signs of end-of- 
dose deterioration and morning bradykinesia are generally the first clinical changes to appear. The "off "phase is defined as a lack of mobility (bradykinesia, akinesia, or stiffness), while during the "on" phase, the patient responds to L-DOPA, regardless of the presence of dyskinesias [1-4]. Motor fluctuations, the primary side effect of long-term L-DOPA therapy, may be a consequence of the short half-life of oral L-DOPA. Likewise, several alterations of dopamine receptors have been described. These are mainly associated with the involved molecular signalling pathways. The modification of L-DOPA dosage and changes to its pharmaceutical formulation are the most common therapeutic strategies used to manage these motor fluctuations [8-13]. Increasing the half-life of L-DOPA helps to prevent fluctuations in plasma L-DOPA levels and increases the amount of L-DOPA available to the brain.

This objective can be achieved by administering a COMT inhibitor together with L-DOPA / DDI therapy [14-19].

COMT inhibitors allow a more continuous level of L-DOPA in the brain due to reduced fluctuation of plasma levels and more constant central dopaminergic stimulation, thus reducing the levels of 3-O-methyl-levodopa which may compete with L-DOPA at the blood-brain barrier. [16-19].

It has been proposed that COMT inhibitors with limited access to the brain may serve as effective adjunctive therapy for $\mathrm{PD}$, avoiding potential undesired central side effects. ENT is currently the standard COMT inhibitor used in the treatment of PD. It is well tolerated and reduces the total "off " time by an average of 45 minutes daily [17-20]. However, it is less efficacious than TOL, another COMT inhibitor. Unfortunately, TOL may cause liver toxicity [21]. Consequently, there exists a need to develop new COMT inhibitors with improved therapeutic and safety profiles. [1921].

In the present manuscript, the authors discuss the preclinical development of OPC, a potent peripheral, selective and reversible inhibitor of COMT, approved in Europe as an adjunct therapy with the administration of L-DOPA / DDI inhibitors in adult patients with PD who present with end-of-dose motor fluctuations and, therefore, cannot be stabilized with L-DOPA/ DDI inhibitors [22]. 


\section{Discovery strategy and preclinical development}

\subsection{Introduction to Opicapone: the rationale for use in Parkinson's disease}

OPC (Marketed as Ongentys, the drug was synthesized and marketed by BIAL Portela \& Ca, S.A. Portugal) received approval for marketing authorization from the European Medical Commission in June 2016 as adjunctive therapy to L-DOPA/DDI in adult patients. The Food and Drug Administration (FDA) accepted the new drug application of OPC as a potential add-on therapy to L-DOPA/carbidopa for PD in April 2020. OPC is a novel third generation, highly potent, once daily and effective COMT inhibitor that optimizes the pharmacokinetics and bioavailability of LDOPA therapy. Given the safety issues with the use of TOL, OPC may be considered a safer alternative for PD patients that have not responded to treatment with other COMT inhibitors [1422].

Until recently, TOL and ENT were the only COMT inhibitors commercially available COMT inhibitors. In clinical practice, TOL was the first used, but it was withdrawn from Europe in 1998 because there were reported cases of liver damage [23]. Nevertheless, it was reincorporated in European clinical practice with a mandatory control during the first year of treatment of the liver function.

TOL is currently administered in PD treatment, at a dose of $100 \mathrm{mg}$ three times a day, for patients who have not been treated with ENT. ENT is better tolerated than TOL and is administered as a supplement to each daily dose of L-DOPA / DDI [17-20]. This compound extends the half-life of L-DOPA, increasing the bioavailability of the drug and prolonging its effect. While it inhibits COMT centrally, ENT acts peripherally and produces a potent and reversible inhibition [14-20].

\subsection{Opicapone: mechanism of action}

OPC [2,5-dichloro-3-(5-(3,4-dihydroxy-5-nitrophenyl)-1,2,4-oxadiazol-3-yl)-4,6dimethylpyridine 1-oxide], formerly known as BIA 9-1067, is a peripheral, selective and 
reversible inhibitor of COMT (Figure 1). OPC inhibits with a high affinity binding that results in a slower rate of complex dissociation and a longer and more constant duration of action in vivo (> 24 hours) [23]. This enzyme catalyzes the transfer of a methyl group from S-adenosyl-Lmethionine to substrates with a catechol group, resulting in the product of O-methylation and a stoichiometric amount of 5-adenosyl homocysteine [24, 25]. This reaction takes place in the presence of the divalent cation $\mathrm{Mg}^{2+}$. Although the existence of O-methylation processes in animal tissues were observed for the first time in 1951, this enzyme was not described until 1958, when it was partially purified [15]. The same year, Axelrod and co-workers showed that this enzyme catalyzes the O-methylation of catecholamines such as dopamine and adrenaline. Studies carried out thereafter showed that COMT plays an important physiological role in the extraneuronal inactivation of endogenous catecholamines [26].

In the presence of a DDI,COMT becomes the main metabolizing enzyme of L-DOPA, catalysing its conversion to 3-O-methyldopa (3-OMD) in the brain and the periphery. In patients taking LDOPA and a peripheral DDI, such as carbidopa or benserazide, OPC increases plasma L-DOPA levels $[25,27]$. The recommended dose of OPC is $50 \mathrm{mg}$ orally, given once daily at bedtime, at least one hour before or after the combination of L-DOPA [23,27]. It may be necessary to adjust the dose of L-DOPA during the initial course of treatment with OPC, by either extending the treatment intervals or decreasing the dose. Although hepatotoxic effects have not been demonstrated, it is not recommended to administer OPC to patients with severe liver impairment, and caution should be taken with moderate hepatic impairment [22, 23, 25, 27]. Precaution is also recommended in aged patients.

\subsection{Pharmacodynamics of Opicapone}

In a set of preclinical research studies in vitro and in vivo, OPC was examined for its mechanism of action, the affinities of binding to peripheral COMT and the effect on the availability of LDOPA after concomitant use with L-DOPA and DDI [28-30]. Experiments were performed in rats and monkeys. The first preclinical research studies of OPC as a COMT inhibitor in PD 
treatment were published by Kiss and colleagues in 2010 [19]. They reported that dose-dependent inhibition of rat liver COMT is determined $3 \mathrm{~h}$ after oral administration of increasing doses (from 0.03 to $10 \mathrm{mg} / \mathrm{kg}$ ) of OPC in rats. In addition, OPC increased 2-fold the plasma L-DOPA levels at $2 \mathrm{~h}$. This increase was constant and maintained over a $24 \mathrm{~h}$ period. The ability of OPC to deliver a consistent increase in L-DOPA levels over time was related to a prolonged peripheral COMT inhibition, suggesting a substantial advantage over the other COMT inhibitors that were on the market.

Moreover, it was reported that the administration of OPC in rats $(3 \mathrm{mg} / \mathrm{kg}$, p.o.) produced an $80 \%$ COMT inhibition in liver and kidney from 1 to $8 \mathrm{~h}$ post-administration. The enzyme reached 70 $86 \%$ of control values at $48 \mathrm{~h}[28-30]$.

In rats, when OPC (3mg/kg, p.o.) was administered 2 and $24 \mathrm{~h}$ prior to L-DOPA/benserazide $(12 \mathrm{mg} / \mathrm{kg}, 3 \mathrm{mg} / \mathrm{kg})$, there resulted a sustained increase, for at least $24 \mathrm{~h}$, of L-DOPA bioavailability by 1.9- and 1.3-fold [28].

Therefore, OPC increased peripheral and central bioavailability of L-DOPA in rats. Gonçalves and colleagues studied a $30 \mathrm{mg} / \mathrm{kg}$ dose in rats, reporting that the effect of COMT inhibition produced by OPC was rapid, within 1 h post-dosing, and nearly complete, with 97\% inhibition, in erythrocytes, liver and kidney. In addition, authors compared the duration of action of the three COMT inhibitors OPC, TOL and ENT [30]. In the case of OPC, at $24 \mathrm{~h}$ following oral administration, greater than $50 \%$ of COMT activity was still inhibited. In the case of TOL and ENT, at $12 \mathrm{~h}$ following administration, the percentage of liver COMT inhibition was $50 \%$ and $15 \%$, respectively.

It has been also reported that OPC metabolites, BIA 9-1079, BIA 9-1103 and BIA 9-1104 were also shown to actively inhibit rat liver COMT, whereas BIA 9-1100 and BIA 9-3752 were inactive [23].

Bonifácio and colleagues investigated the effect of OPC on brain availability and disposition of L-DOPA by means of simultaneous microdialysis of the substantia nigra and other brain areas in cynomolgus monkey [31]. They found that erythrocyte COMT activity that was inhibited by $75 \%$. In addition, in the OPC-treated substantia nigra dialysates, there was a 1.4-fold increase in L- 
DOPA levels. At the peripheral level, animals treated with intracerebroventricular (ICV) OPC significantly increased two-fold the plasma L-DOPA bioavailability. Kitajima and colleagues examined the effect of acute and repeated administration of OPC (1, 10 and $100 \mathrm{mg} / \mathrm{kg})$ on erythrocyte COMT activity in cynomolgus monkey $[32,33]$. Their results showed that COMT activity was significantly inhibited by OPC at 10 and $100 \mathrm{mg} / \mathrm{kg}$, with a COMT inhibition mean of $76.4 \%$ and $93.2 \%$ from baseline, respectively, and a maximal effect at $2 \mathrm{~h}$ with both doses. After 24 hours, COMT activity was evaluated again and results exhibited an inhibition percentage of $42.6 \%$ and $60.2 \%$ in both groups, respectively $[32,33]$.

In preclinical models of $\mathrm{PD}$, using 1-methyl-4-phenyl-1,2,3,6-tetrahydropyridine (MPTP) in cynomolgus monkeys, Bonifacio and colleagues reported that OPC improved the effects of LDOPA/Benserazide therapy, reducing MPTP-induced PD-like symptoms, increasing L-DOPA in plasma and reducing plasma 3-OMD levels and erythrocyte COMT activity [34, 35] (Table 1). Moura and colleagues demonstrated that in a hemi-parkinsonian model of PD, achieved through injection of 6-OHDA, OPC administration to rats ( $3 \mathrm{mg} / \mathrm{kg}$, p.o.) once daily for three consecutive days prior to L-DOPA/ benserazide produced an improvement in rotational behaviour [36] (Table 1). This effect was also associated with a significant peripheral COMT inhibition in OPC treated animals.

\subsection{Pharmacokine tic profile}

Preclinical studies reported that following oral administration of OPC, it was rapidly absorbed, reaching its $\mathrm{T}_{\max }$ in less than 4 hours. The drug possesses a short half-life in plasma and binds strongly to rat plasma proteins $(99.7 \%)$.

The prolonged inhibition of COMT, observed in the rat and the cynomolgus monkey after OPC administration was not, however, due to the presence of the compound in the circulation $[23,27$ 30].

In addition, OPC is completely metabolized in the liver 8 hours after its oral administration, yielding several inactive metabolites; biotransformation is mainly via conjugation (sulphation, 
glucuronidation and methylation) [23]. Several OPC metabolites in rodents have been reported, including BIA 9-1100 (methylated metabolite, inactive), BIA 9-1103 (sulfated metabolite, inactive), BIA 9-1106 (glucuronide metabolite, inactive) and its active metabolite BIA 9-1079 (an amine N-oxide reduced form of OPC) [23, 37]. Rat plasma levels of BIA 9-1079 remained in a high relative percentage (33.6-56.5\%) of total OPC, and for this reason the active metabolite can play an important role in contributing to the pharmacological therapeutic activity of OPC in rodents [38-46].

However, clinical studies reported that the percentage of the active metabolite BIA 9-1079 was less than $15 \%$ of total OPC derivatives [47-50]. Thus, authors suggest that the potential contribution of the active metabolite to the therapeutic effect of OPC should be much lower than expected for drugs.

The most abundant plasma peaks after a single dose of $100 \mathrm{mg}$ of ${ }^{14} \mathrm{C}-\mathrm{OPC}$ are the metabolites BIA 9-1103 (sulfate) and BIA 9-1104 (methylated), representing 67.1 and 20.5\% of inactive metabolites [23, 27]. Other metabolic pathways include glucuronidation, methylation and reduction. The recommended therapeutic dose in humans is $50 \mathrm{mg}$ OPC once daily [23, 27].

Other metabolites were not found in quantifiable concentrations in the majority of the plasma samples collected during a clinical mass balance study. BIA 9-1100 and BIA 9-3752 were demonstrated to be inactive. From the dose-response curves, IC50 values for rat liver S-COMT inhibition of $224 \mathrm{nM}(0.09 \mu \mathrm{g} / \mathrm{ml}), 429 \mathrm{nM}(0.17 \mu \mathrm{g} / \mathrm{ml})$ and $128 \mathrm{nM}(0.05 \mu \mathrm{g} / \mathrm{ml})$ were determined for OPC, BIA 91079 and BIA 9-1104, respectively [23].

Gonçalves and colleagues reported on pharmacokinetic studies of OPC following administration of single oral doses $(30,60$ and $90 \mathrm{mg} / \mathrm{kg}$ ) to Wistar rats [38, 43-45]. In the study, blood samples were collected at different times $(0.5-24 \mathrm{~h})$. The plasma levels of OPC and its active metabolite (BIA 9-1079) were quantified by high-performance liquid chromatography (HPLC) with a diodearray detector. OPC administration to rats caused a systemic increased exposure in a doseproportional manner and, considering the $\mathrm{AUC}_{0^{-2}-2 \mathrm{~h}}$ values achieved, BIA 9-1079 represented $53.7 \%(30 \mathrm{mg} / \mathrm{kg}), 56.6 \%(60 \mathrm{mg} / \mathrm{kg})$ and $33.6 \%(90 \mathrm{mg} / \mathrm{kg})$ of total OPC derivatives. 
Loureiro and colleagues, evaluated the metabolism of OPC in the cynomolgus monkey and the Wistar rat after intravenous $(1 \mathrm{mg} / \mathrm{kg})$ and oral administration $(10 \mathrm{mg} / \mathrm{kg})$ of $\left[{ }^{14} \mathrm{C}\right]-\mathrm{OPC}$. The studies indicate that OPC was rapidly absorbed and distributed throughout the body in both species [44]. Likewise, after 5-7 days administration of $\left[{ }^{14} \mathrm{C}\right]-\mathrm{OPC}$ in rats, more than $89.0 \%$ of related radioactivity was excreted.

More than $68 \%$ of OPC and metabolite radioactivity was excreted via the biliary route in animals, and the same results were reported in humans. In monkeys, very low levels (less than 10\%) were excreted by the kidneys [23. 44]. In rats and humans, renal excretion accounted for approximately $10 \%$ and $12 \%$ of total excretion, respectively $[44,48]$. This percentage of BIA 9-1106 in the urine suggests that kidney is not the main route of excretion for OPC and other metabolites. The authors concluded that OPC is mostly excreted via the biliary route. In Cynomolgus monkey and Wistar rats, faecal route is the main route of excretion [23]. Furthermore, there are differences between the formations of OPC metabolites between species [23].

\subsection{Toxicologic profile}

One of the major concerns with the use of COMT inhibitors is hepatoxicity caused by TOL administration [21]. The use of TOL requires liver function monitoring, due to the risk of liver toxicity. At in vitro level, some pre-clinical studies demonstrated that COMT inhibitors exhibited several hepatotoxicity effects on mitochondrial membrane and cellular adenosine triphosphate (ATP) content [19, 23, 28, 29].

By measuring mitochondrial membrane potential (MMP) and ATP production in human primary hepatocytes, a 50\% decrease in both MMP $(181 \mu \mathrm{M})$ and ATP $(98 \mu \mathrm{M})$ was noted following 24 $\mathrm{h}$ incubation with OPC $[19,28]$. This finding suggested that OPC was a safe compound, since compared with TOL and ENT, it caused less reduction in both MMP and ATP levels. ENT depleted ATP with an $\mathrm{IC}_{50}$ of $45 \mu \mathrm{M}$ and decreased the MMP with an IC50 of $4 \mu \mathrm{M}$ and TOL 25 $\mu \mathrm{M}$ and $29 \mu \mathrm{M}$ respectively [38] (Table 1). 
Thus, these data give support to the low hepatotoxic effects of OPC. Taking into account these data, no liver toxicity concerns were expected with the dosing regimens tested in preclinical studies.

The same research team examined the toxicity of the three COMT inhibitors in HepaRG cells, measuring ATP depletion (indicator of cell viability), caspase 3/7 activation (indicator of apoptosis), glutathione depletion (indicator of reactive metabolite formation) and loss of the mitochondrial membrane potential (mitochondrial dysfunction) [51]. In HepaRGcells, increasing concentrations of OPC, ENT or TOL produced a progressive decrease in MMP, with IC50 values of $0.5 \mathrm{mM}, 1.0 \mathrm{mM}$ and $0.1 \mathrm{mM}$, respectively. Determination of ATP content in these samples revealed no toxicity effect at any of the tested concentrations for OPC or ENT, but at $200 \mu \mathrm{M}$ a decrease in ATP content was observed for TOL [51]. COMT inhibitors at $30 \mu \mathrm{M}, 100 \mu \mathrm{M}$ or 200 $\mu \mathrm{M}$ did not increase caspase 3 activity. In terms of oxidative stress, the only significant decrease in glutathione levels occurred at $200 \mu \mathrm{M}$ (28\% of control values) of TOL. Since OPC did not decrease ATP cellular levels or increase apoptosis, nor did it lead to oxidative stress in human cell line HepaRG [23, 51], it was deemed to be a safe compound in terms of liver toxicity.

Lopes and collaborators confirmed the results obtained at pre-clinical studies in a multicentre, double blind, randomised, placebo- and active-controlled study (BIPARK II and I). In this study 509 total subjects were treated with OPC ( 25 or $50 \mathrm{mg}$ ) and 257 with placebo. They concluded that there were no relevant differences between OPC and placebo group in the parameters associated with liver laboratory toxicity [52-54]. Moreover, no significant cardiovascular effects were observed in dogs, with the exception of a slight shortening in the QT interval observed at $600 \mathrm{mg} / \mathrm{kg}$. No effect on action potential parameters was noted.

In vitro studies showed low cardiac toxicity with high dose OPC $[23,55,56]$. Pinto and colleagues evaluated the effects of OPC on cardiac repolarization in healthy adult volunteers. At the evaluated doses of OPC in the study (50 and $800 \mathrm{mg}$ ), no adverse effects on electrocardiogram intervals were observed $[55,56]$. Thus, OPC has a negligible cardiac toxicity risk in preclinical and clinical studies [23]. 
Castro-Caldas and colleagues reported data from human clinical studies showing that OPC adverse events induced by COMT inhibitors are associated with an increase in dopaminergic and non-dopaminergic stimulation [57]. The most common side effects were due to dopaminergic potentiation, since the main objective of OPC is to increase the bioavailability and supply of dopamine to the brain. The most frequent adverse events associated with OPC treatment, dyskinesias and nausea, were found to be mediated by dopaminergic stimulation, independent of dose, and tended to decrease over time. Side effects could be minimized by increasing dose intervals or by reducing the total dose of L-DOPA. The authors concluded that OPC shows a favourable safety profile, it is well-tolerated and adverse reactions are similar to those described for ENT [57].

In summary, preclinical studies suggest that OPC is a safe and well-tolerated drug which possesses a reduced risk of hepatic and cardiac toxicity, and adverse reactions are in line with those described for ENT [23].

\section{Clinical findings}

Two-phase III clinical trials (BIPARK-I, also known as BIA-91067-301, and BIPARK-II, also known BIA-91067-302). Multicentre, randomized, double-blind, placebo-controlled trials were presented for drug approval [58-68].

The European Commission approved OPC under the brand name Ongentys ${ }^{\circledR}$, an adjunct therapy to L-DOPA/DDI in adult patients with PD and end-of-dose motor fluctuations which cannot be stabilized on L-DOPA/DDI alone. [23]. BIPARK-I evaluated the efficacy and safety of OPC at 5, 25 and $50 \mathrm{mg}$, administered once daily, in comparison with ENT (200 mg) or placebo [58, 62, $65,67,68,69$ ] (Table 2). OPC $50 \mathrm{mg}$ once a day (QD) achieved the same therapeutic effects as ENT, and was found to be superior to placebo $[58,69]$.

The study included 600 patients from 106 study centers in Europe. Patient criteria were as follows: The patients were aged between 34 and 83 years old and carried the diagnosis of idiopathic PD for a minimum of 3 years. In the "on" phase, their modified Hoehn and Yahr score was of $\leq 3$. All 
subjects had received optimal treatment with L-DOPA and had been clinically stable for at least 4 weeks. Signs of end-of-dose deterioration were present for at least 4 weeks, with an average of daily "off "time of $1.5 \mathrm{~h}$, excluding AM periods prior to the first daily dose. Finally, subjects were required to demonstrate an ability to keep a precise 24-hr diary [58].

The study showed that the OPC-treated patients experienced an increase in average total "on "time of 119 minutes, as compared with 47.1 minutes in the placebo group, a statistically significant difference [58, 67, 69]. Most of the time gained referred to the period in which the patient experienced non-problematic dyskinesia, without a significant gain in the "on "time with problematic dyskinesia [58] (Table 2).

BIPARK-11 (BIA-91067-302) included 427 patients, randomly assigned to OPC 25mg and 50 mg treatment dose groups [59]. At the end of this period, subjects entered an open phase of OPC treatment of 67 weeks. Of 427 subjects involved in this study, 376 patients completed the double blind phase. The 50mg group had a greater number of "off "and "on" time responders compared with placebo. At $25 \mathrm{mg}$, OPC effectively increased the percentage of responders in "on" time. However, with regards to "off "time response, the $25 \mathrm{mg}$ OCP group fared no better than placebo. With regards to the absolute change in "on" time, significant differences were observed in the $50 \mathrm{mg}$ treatment group compared to placebo (52.59 $\mathrm{min})$. No significant differences were noted between treatment and control groups when considering "on" time without dyskinesia or troublesome dyskinesia [59

The most frequently observed adverse effects related to treatment were: dyskinesia $(7.6 \%-24 \%$ in OPC, $8.2 \%$ in ENT, $4.1 \%-8.1 \%$ in PLA), L-DOPA constipation $(0.9 \%,-6 \%$ in OPC, $4.1 \%$ in ENT, $1.5 \%-2.5 \%$ in placebo), insomnia (1.3\% - 8\% in OPC, $5.7 \%$ in ENT, $0.8 \%-2.2 \%$ in PLA), dry mouth $(1.6 \%-10.4 \%$ in OPC, $1.6 \%$ in ENT, $0.7 \%-1.7 \%$ in placebo), increased creatine phosphokinase (CPK) (0.9\% -8\% in OPC, $0.8 \%$ in ENT, $0 \%-3.7 \%$ in PLA), and dizziness (1.6\% $-5 \%$ in OPC, $4.1 \%$ in ENT, $0,8 \%-1.5 \%$ in PLA) $[23,27,59,70-74]$.

OPC treatment effectively reduced "off " time and increased "on " time without increasing the frequency of dyskinesia, and this benefit was maintained for_at least_1 year of therapy without 
increasing the dose of L-DOPA $[23,27,53,59]$. 50mg OPC daily is thus considered the most efficacious dose, providing an average of one additional hour of "off "time reduction [53].

\section{Post-launch of Opicapone}

In June 2016, the EMA approved the new drug Ongentys ${ }^{\circledR}$ (OPC) for oral treatment of PD adult patients with motor fluctuations [23, 27]. OPC has been launched in the United Kingdom, Germany, Portugal and Spain, and it is expected that, between 2020 and 2021, it will be introduced in other European countries as well as the United States, China and Japan. In February 2017, BIAL and Neurocrine Biosciences, Inc. announced that they had entered into a licensing agreement for the development and commercialization of OPC in North America. Recently, the FDA accepted OPC for PD (co-adjuvant) as a complementary treatment to L-DOPA / carbidopa in patients with "off " episodes. Likewise, a 12-month review process was established which will be detailed in, the Prescription Drug User Fees Act starting April 2020.

In April 2013, Ono Pharmaceutical entered into a license agreement with BIAL for the Japan market. In February 2019, Ono Pharmaceutical submitted an application for manufacturing and marketing approval of OPC. In January 2018, BIAL and Jiangsu Wanbang Biopharmaceutical Group Co., Ltd. announced that they had entered into a license agreement for the commercialization of OPC in China. OPC is available as hard capsules (25 and $50 \mathrm{mg}$ ), and is taken once daily at bedtime, at least_ 1 hour before or after L-DOPA.

\subsection{Post-marketing data}

A phase 4 clinical trial (NCT02847442, OPTIPARK) is currently ongoing in the European Union, aiming to explore the potential safety and tolerability of OPC $50 \mathrm{mg}$ during a period of 3 months among 518 PD patients with end-of-dose motor fluctuations. This is a prospective, open-label, single-group, multi-centre trial in PD patients with wearing-off motor fluctuations. However, still long-term studies are still needed to ensure the safety profile and demonstrate the advantage of OPC over the other COMT inhibitor drugs in the pharmaceutical market for PD treatment. 


\subsection{Safe ty and pharmacovigilance}

The safety, efficacy and tolerability of OPC was evaluated over the course of a year in the open extension phase $[53,75]$ (Table 2). To perform this study, patients who completed the double-blind part of the BIPARK II study were enrolled in an open 1-year extension phase. Pooled analyses of the double-blind and open-label stages of BIPARK I and II have been carried out. During this extension phase, two groups were studied. Patients that had previously taken placebo or ENT were started on 50mg OPC. The second group, having previously taken 50mg OPC, were continued on the same dose $[53,75]$.

The extension period data were analysed with the aim of elucidating the long-term effects of OPC. To date, two studies based on the extension phase data have been published. Looking at data generated from both the double-blind and the open BIPARK 1 and 2 studies, Lees and coworkers aimed to evaluate the safety and tolerability of adjunct OPC (25 and 50mg) in PD patients treated with L-DOPA.

The majority of adverse events were mild or moderate in severity and generally transient and manageable, as indicated by the low interruption rate. Long-term use of OPC, once a day for 1 year at a dose of $25 \mathrm{mg}$ or $50 \mathrm{mg}$, does not appear to result in any new or unexpected safety problems.

At 50mg OPC daily, no evidence for hepatic injury was noted. Furthermore, side effects that had been reported with both TOL and ENT, such as severe diarrhoea, were not found. The percentage of patients who interrupted the treatment due to adverse events mediated by OPC was low and similar in all treatment groups [53].

In a similar study carried out by Ferreira and colleagues, the same population groups were evaluated at doses of both 25 and 50mg OPC. Significant improvements in motor fluctuations were noted in the study groups, without a significant increase in dopaminergic side effects such as dyskinesia [75]. 
In addition, it was observed that when switching from placebo to OPC in the extension phase, the "off" time was reduced by an average of 51.1 minutes. During the double-blind phase, a one-year follow-up showed that patients previously treated with OPC at 25 and 50mg demonstrated an average "off" time reduction of 35.1 minutes and 58.1 minutes, respectively, with a total "off "time reduction average of nearly 2 hours per day.

With regard to "on" time, in the patients originally receiving placebo, there was an average increase of 52.5 minutes, but no change in those patients that initially received 50mg OPC.

The most frequent side effect observed was dyskinesias at $16 \%$ in the treatment group.

\subsection{Studies on market and main competitors}

Currently, two COMT inhibitors, ENT and TOL, remain on the market for the treatment of PD. While ENT is a safe product which is given several times a day, TOL requires close monitoring due to the potential risk of hepatotoxicity. However, TOL is more potent than ENT.

OPC is currently marketed under the Ongentys ${ }^{\circledR}$ brand name in the United Kingdom, Germany, Spain, Italy and North America. Under the terms of the agreement, Neurocrine will be responsible for development and commercialization of OPC in the United States and Canada. Bialis launched OPC in EU market in 2018. In its first year, sales of OPC in the PD market are projected to have reached \$3.7million. By 2022, global sales are projected to increase to $\$ 68.6$ million $[76,77]$.

However, there are some drawbacks which could slow down the OPC growth in pharmaceutical market of PD treatments such as competition with Stalevo ${ }^{\circledR}$.

Stalevo $^{\circledR}$ is a combination of L-DOPA / carbidopa in the same pill with production costs that are lower than OPC.

\section{Conclusions}

PD is a devastatingly progressive disease with only symptomatic therapy currently available. OPC is a new and potent highly selective peripheral COMT inhibitor (Figure 1). OPC has a high 
binding affinity, which results in a slow rate of dissociation of the complex and a long duration of action that allows once daily administration [78]. Preclinical studies reported that OPC, compared to other COMT inhibitors, is safe and the observed adverse effects are lower, especially with respect to hepatoxicity and cardiotoxicity [23-36] (Table 1).

In addition, in animal studies it has been observed that the active metabolite BIA 9-1079 contributes to the therapeutic effect, which does not occur in humans.

Moreover, preclinical data in rat and monkey models demonstrate that OPC enhances significantly the bioavailability of L-DOPA [23, 40-45]. In both MPTP and 6-OHD-induced PD animal models a significant improvement of movement-related disease symptoms can be demonstrated when OPC is used as an adjunct with L-DOPA / DDI. Preclinical results indicate that OPC is an effective compound with suitable pharmacokinetics, a potent inhibitor of COMT which produces few side effects.

Results of two-phase III studies conducted in patients with PD demonstrated a significant reduction in "off " time following administration of $50 \mathrm{mg}$ OPC as well as increased "on" time (Table 2). The BIPARK-I study also showed greater efficacy of OPC over ENT [58]. OPC 50 mg appears to demonstrate a greater effect on "off " time reduction suggesting a potentially greater clinical benefit.

The BIPARK-2 study confirms the presence of fewer adverse effect such as diarrhoea, a side effect characteristic of COMT inhibitors [59]. As suggested by the low rate of patient withdrawal due to side effects, OPC appears to be well tolerated.

When compared to other COMT, OPC is administered once daily, one hour before or after administration of L-DOPA, while ENT is administered up to 8 times a day along with L-DOPA. Inasmuch as L-DOPA may be taken multiple times a day, the QD dosing of OPC does not necessarily simplify the dosing regimen. Presently an ENT/L-DOPA/DDI inhibitor combination tablet is available, facilitating dosing [79-82]. TOL is administered three times a day, but due to potential hepatotoxicity, TOL is recommended only for those patients unable to tolerate or have poor clinical response to ENT or OPC. In patients with mild hepatic impairment, OPC may be administered although dosing adjustments may need to be made. 
Given the safety issues with the use of TOL, OPC may be considered a safer alternative in patients who have not responded to treatment with ENT.

\section{Expe rt opinion}

Ideal treatment of PD would be to modify the course of the illness, but currently no such treatment exists.

Different strategies have been proposed to treat PD. For example, based upon the observation that mitochondrial dysfunction and oxidative damage may play a role in the pathogenesis of $\mathrm{PD}$, a study (clinicaltrials.gov Identifier: NCT00740714) examined the effectiveness of treatment with 2400mg CoQ10 in idiopathic PD. However, in phase III clinical trials, although was safe and well tolerated by patients, patients did not show significant clinical improvement [83].

It has been observed that the CNS of PD patients contain abnormal clumps of the protein alphasynuclein. It has been proposed that this protein forms an extracellular neurotoxin implicated in the pathogenesis of PD. A monoclonal IgG1 antibody, PRX002 / RG7935, has been designed to attack the $\mathrm{C}$-terminal of neurotoxic aggregate forms of $\alpha$-synuclein and reduce their levels in the brain [84]. A phase 1 clinical trial utilizing intravenous infusions of PRX002V (ClinicalTrials.govIdentifier:NCT02157714) has shown acceptable safety profiles, and a phase 2 trial is now underway, with completion in 2021 (NCT03100149) [84, 85].

Another strategy used to modify or delay the disease has been the use of neurotrophic growth factors such as glial cell-line-derived neurotrophic factor (GDNF) and neurturin (NTN), which are members of the transforming growth factor $\beta$ superfamily [86]. The objective of these strategies is to restore the viability of degenerated neurons. A clinical trial (ClinicalTrials.gov, number NCT00985517) has been conducted based on the direct administration of the gene therapy of an AAV2-neurturin vector (CERE-120) in the brain (to the putamen plus substantianigra) [8688]. However, the phase 2 was discontinued because the compound did not demonstrate statistically significant efficacy for an improvement in patient scores according to the Unified Parkinson's Disease Rating Scale. Glial derived neurotrophic factor (GDNF) was also developed as a therapy that would modify PD [89]. However, the results of the clinical trial were not 
satisfactory, but the authors suggest hopes for longer GDNF treatments that it is possible to restore damaged cells in PD $[89,90]$. Trophic factors may represent a future PD treatment, however this approach has not met with great success [88].

Since there is no cure for $\mathrm{PD}$, the objective of the treatment consists of controlling the motor symptoms primarily through administration of L-DOPA/DDI and improving the quality of life of patients [2-4]. Although L-DOPA improves the control of PD symptoms, it is not able to halt disease progression, and, furthermore, the beneficial effects of L-DOPA eventually wear off, leading to worsening of symptoms and the appearance of motor fluctuations in patients.

In the presence of a DDI inhibitor, COMT becomes the main metabolizing enzyme of L-DOPA, catalysing its conversion to 3-O-methyldopa (3-OMD) not only in the brain but also in the periphery. OPC is a peripheral, selective and reversible inhibitor of COMT with a high binding affinity that results in the slowing in the rate of complex dissociation leading to a long and constant duration of action in vivo (> 24 hours). In patients taking L-DOPA and a DDI, OPC significantly improves PD symptomology increasing plasma L-DOPA levels in the CNS. Thus, OPC provides a suitable strategy that could well accommodate the therapeutic needs of PD patients.

An important challenge in PD treatment is to minimize the "off " time and to increase the "on" time, a property related to the efficacy and the pharmacokinetic properties of the drug. In the absence of disease-modifying treatments, the therapeutic strategy is based on improving treatment with L-DOPA by enhancing the "on " phenomenon and decreasing the "off" effect. As demonstrated by clinical trials, the latter has been ameliorated with the administration of OPC [53-75].

Before OPC approval for the treatment of PD, only two COMT inhibitors were available for clinical L-DOPA/DDI association for the management of end-of-dose motor fluctuations: TOL and ENT. TOL was the first COMT inhibitor to be commercially available and it is a more potent COMT inhibitor than ENT, both in the periphery and CNS [20-23]; however, it has also been associated with severe liver injury. For this reason, it's administration requires continuous liver 
function monitoring and must be restricted to patients who have failed to respond, or those patients that are intolerant to other COMT inhibitors. Conversely, ENT acts only in the periphery and is safer than TOL but has limited efficacy as well as a low to moderate oral bioavailability, which requires frequent dosing $[76,77]$.

Accordingly, OPC represents an improvement in the treatment of PD compared to the other COMT inhibitors. The beneficial effects of OPC in PD therapy are currently thought to be due to a) dosing regimen, since it is administered once a day, b) lack of hepatotoxicity compared with TOL (ENT is not associated with hepatotoxicity, therefore, it does not require liver monitoring), c) higher mean reduction of "off" time, with an average reduction of $116.8 \mathrm{~min}$ at the therapeutic dose of 50mg OPC.

Furthermore, ENT has the disadvantage of low oral bioavailability with a shorter duration of action as well as being less potent.

At the recommended therapeutic dose of $200 \mathrm{mg}$, ENT therefore has limited clinical efficacy_[79, 80]. OPC meets the need for a safer, more potent and long-acting COMT inhibitor [80]. As a selective COMT, reduction in "off " time provides a new strategy that could well accommodate the therapeutic needs of PD patients. A small molecule such as OPC demonstrates proven

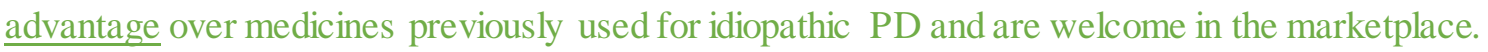
Of course, OPC represents symptomatic treatment of PD; a curative or preventative approach remains elusive.

In conclusion, despite its limitations, OPC constitutes a paradigm shift in the treatment of PD. It may represent a starting point for both a better understanding of PD's pathogenesis as well as the development of new types of medical and pharmaceutical interventions to improve care for PD patients.

However, improvements in the design of COMT inhibitors should still be pursued, including, for example, an improved half-life and low toxicity whilst maintaining or increasing drug efficacy. COMT inhibitors currently marketed (with the exception of TOL) are intended to act exclusively in the inhibition of peripheral COMT. We strongly believe that it would be of interest for new drugs to also exert a centrally acting COMT inhibitor effect. 


\section{Acknowledgments}

William Scott Allyn MD, for reviewing the manuscript, corrections, and constructive criticism.

\section{Funding}

The Spanish Ministry of Science and Innovation SAF2017-84283-R, CB06/05/0024 (CIBERNED), the European Regional Development Funds supported this work. Research team from UB and URV belongs to 2017SGR625 from Generalitat de Catalunya. PRM is supported by grants 2015/26084-1 and 2017/13224-5, Sao Paulo Research Foundation (FAPESP) - Brazil.

\section{CONFLICT OF INTERESTS}

None of the authors have any conflicts of interest including any financial, personal or other relationships with other people or organizations. All authors have reviewed the contents of the manuscript being submitted, approved its contents and validated the accuracy of the information.

\section{ORCID}

Antoni Camins http://orcid.org/ 0000-0002-1229-5956

\section{REFERENCES}

1. Poewe W, Seppi K, Tanner CM, et al. Parkinson disease. Nat Rev Dis Primers 2017;3:17013.

2. Balestrino R, Schapira AHV. Parkinson disease. Eur J Neurol. 2019 Oct 20.

3. Baizabal-Carvallo JF, Hallett M, Jankovic J. Pathogenesis and pathophysiology of functional (psychogenic) movement disorders. Neurobiol Dis. 2019;127:32-44.

4. Simon DK, Tanner CM, Brundin P. Parkinson Disease Epidemiology, Pathology, Genetics, and Pathophysiology. Clin Geriatr Med. 2020 Feb;36(1):1-12.

5. Cheong SL, Federico S, Spalluto G, Klotz KN, Pastorin G. The current status of pharmacotherapy for the treatment of Parkinson's disease: transition from single-target to multitarget therapy. Drug Discov Today. 2019;24:1769-1783.

6. Zesiewicz TA. Parkinson Disease. Continuum (Minneap Minn). 2019 ;25:896-918.

7. Lang A, Lees A. Management of Parkinson's disease: an evidence based review. Mov Disord. 2002;17 Suppl 4:S1-166.

8. Gupta HV, Lyons KE, Pahwa R. Old Drugs, New Delivery Systems in Parkinson's Disease. Drugs Aging. 2019;36:807-821 
9. Barroso SDS, Lopes LES, Santos KS, Gomes MZ. Technological prospection: patents mapping involving compounds for the treatment of L-DOPA-induced dyskinesias. Expert Opin Ther Pat. 2019;29:979-985.

10. Marino BLB, Souza LR, Sousa KPA, et al. Parkinson's disease: A Review from the Pathophysiology to Diagnosis, New Perspectives for Pharmacological Treatment. Mini Rev Med Chem. 2019 Nov 3.

11. Factor SA, Burkhard PR, Caroff S, et al. Recent developments in drug-induced movement disorders: a mixed picture. Lancet Neurol. $2019 ; 18: 880-890$.

12. Hoy SM. Levodopa/Carbidopa Enteral Suspension: A Review in Advanced Parkinson's Disease. Drugs. 2019; 79:1709-1718.

13. Rascol O, Perez-Lloret S, Ferreira JJ. New treatments for levodopa-induced motor complications. Mov Disord. 2015; 30:1451-1460.

14. Deane KH, Spieker S, Clarke CE. Catechol-O-methyltransferase inhibitors for levodopainduced complications in Parkinson's disease. Cochrane Database Syst Rev. 2004;4:CD004554.

\section{Review on entacapone and tolcapone in treatment of PD patients with motor} fluctuations.

15. Männistö PT, Kaakkola S. Catechol-O-methyltransferase (COMT): biochemistry, molecular biology, pharmacology, and clinical efficacy of the new selective COMT inhibitors. Pharmacol Rev. 1999; 51:593-628.

16. Bonifácio MJ, Palma PN, Almeida L, Soares-da-Silva P. Catechol-O-methyltransferase and its inhibitors in Parkinson's disease. CNS Drug Rev. 2007;13:352-379.

17. Kiss LE, Soares-da-Silva P. Medicinal chemistry of catechol O-methyltransferase (COMT) inhibitors and their therapeutic utility. J Med Chem. 2014; 57:8692-8717.

18. Learmonth DA, Kiss LE, Soares-da-Silva P. The chemistry of catechol-O-methyltransferase inhibitors. Int Rev Neurobiol. 2010; 95:119-162.

19. Kiss LE, Ferreira HS, Torrão L, et al. Discovery of a long-acting, peripherally selective inhibitor of catechol-O-methyltransferase. J Med Chem. 2010; 53:3396-3411. 
20. Entacapone. LiverTox: Clinical and Research Information on Drug-Induced Liver Injury [Internet]. Bethesda (MD): National Institute of Diabetes and Digestive and Kidney Diseases; 2012- 2017 Jul 20.

21. Tolcapone. LiverTox: Clinical and Research Information on Drug-Induced Liver Injury [Internet]. Bethesda (MD): National Institute of Diabetes and Digestive and Kidney Diseases; 2012-2017 Jul 20.

22. Brooks DJ. Safety and tolerability of COMT inhibitors. Neurology. 2004;62(1 Suppl 1): S39-46.

\section{-• Review on safety and tole rability of tolcapone and entacapone.}

23. EMA. Ongentys. Assessment Report. EMA/343011/2016. Avaliable for download at http:/www.ema.europa.eu/ema/Index.jsp?curl=pages/medicines/human/medicines/002790/ human med 001950.jsp\&mid=WC0b01ac058001d124.

24. Palma N, Bonifacio MJ, Loureiro AI, Soares-da-Silva P. Computation of binding affinity of catechol-o-methyltransferase-opicapone complexes. Parkinsonism Rel Disord. 2012; 18S2; S81-S159

25. LeWitt P, Claassen D, Olson K, et al. Once-daily Opicapone increases ON-time in patients with Parkinson's disease: results from two phase 3 studies. Presented at: 2019 American Academy of Neurology Annual Meeting; May 4-10, 2019; Philadelphia, PA.

26. Axelrod J, Laroche MJ. Inhibitor of O-methylation of epinephrine and norepinephrine in vitro and in vivo. Science. 1959;130:800.

27. European Medicines Agency, 2016b. Ongentys (opicapone): EPAR - public assessment report. (Available from http://www.ema.europa.eu/docs/en_GB/document_library/ EPAR__Public_assessment_report/human/002790/WC500209538.pdf (accessed 10.20.16)).

28. Bonifácio MJ, Torrão L, Loureiro AI, et al. Pharmacological profile of opicapone, a thirdgeneration nitrocatechol catechol-Omethyl transferase inhibitor, in the rat. Br. J. Pharmacol. 2015; 172:1739-1752.

- Pharmacological profile of opicapone. 
29. Bonifácio MJ, Torrao MF, Loureiro A, et al. Pharmacological Profile of Opicapone in Wistar rat. Catecholamine Research in the 21st Century. 2014, Page 83.

30. Gonçalves D, Alves G, Fortuna A, et al. A single- and multiple-dose study to investigate the pharmacokinetics and pharmacodynamics of opicapone, a novel COMT inhibitor, in rat. Neuropharmacology. 2017; 125:146-155.

31. Bonifácio MJ, Sutcliffe JS, Torrão L, et al. Brain and peripheral pharmacokinetics of levodopa in the cynomolgus monkey following administration of opicapone, a third generation nitrocatechol COMT inhibitor. Neuropharmacology. 2014; 77:334-341.

32. Kitajima T, Mizote S, Bonifácio MJ, Umemura T, Yoneda K, Moser P, Soares-da-Silva P, Tanaka M. Inhibition of catechol-O-methyltransferase in the cynomolgus monkey by opicapone after acute and repeated administration. Neuropharmacology. 2018; 143:282-288.

33. Kitajima T, Bonifácio M, Moser P, et al. Novel COMT inhibitor opicapone shows sustained inhibition and improved L-DOPA availability in monkeys. International Congress of Parkinson’s Disease and Movement Disorders ${ }^{\circledR}$ Hong Kong October 5-9, 2018; 345.

34. Bonifácio MJ, Sousa F, Soares-da-Silva P. Opicapone improves the effects of 1-dopa on the mptp-induced parkinson's-like syndrome in cynomolgus monkeys. J Neurol Sci. 2017; 381 Suppl 15: 217-218.

- First study demonstrating the efficacy of opicapone in a preclinical PD model

35. Bonifacio MJ, Sousa F, Medakkar S, Vivian J, Soares-da-Silva P. Opicapone improved the effect of L-DOPA on the MPTP induced Parkinson's-like syndrome in cynomolgus monkeys. Parkinsonism Rel Disord. 2016; 22: e149ee192.

36. Moura E, Bonifacio MJ, Soares-da-Silva P. Opicapone improves the levodopa induced rotational behavior in 6-OHDA hemiparkinsonian rats. Parkinsonism Rel Disord. 2016; 22: e149ee192.

37. Loureiro AI, Fernandes-Lopes C, Soares-da-Silva P. Distribution, metabolism and elimination of opicapone in the rat and non-human primates. Parkinsonism Rel Disord. 2016; 22: e149ee192. 
38. Gonçalves D, Alves G, Fortuna A, Soares-da-Silva P, Falcão A. Pharmacokinetics of opicapone, a third-generation COMT inhibitor, after single and multiple oral administration: A comparative study in the rat. Toxicol Appl Pharmacol. 2017; 323:9-15.

39. Gonçalves D, Alves G, Fortuna A, et al. Development of a liquid chromatography assay for the determination of opicapone and BIA 9-1079 in rat matrices. Biomed Chromatogr. 2016; 30:312-322.

40. Bonifacio MJ, Sutcliffe JS, Torrao LS, Brain and peripheral levodopa pharmacokinetics in the cynomolgus monkey following administration of opicapone, a novel catechol-omethyltransferase inhibitor. Parkinsonism Rel Disord. 2012; 18S2; S81-S159.

- The first study evaluating the therapeutic efficacy of OPC in primates

41. Bonifácio MJ, Sutcliffe JS, Torrão L, et al. Effect of Opicapone, a New Catechol-OMethyltransferase Inhibitor, in Levodopa Pharmakokinetics in the Cynomolgous Monkey. Catecholamine Research in the 21st Century, 2014, 79.

42. Bonifácio MJ, Sutcliffe JS, Torrão L, et al. Brain and peripheral pharmacokinetics of levodopa in the cynomolgus monkey following administration of opicapone, a third generation nitrocatechol COMT inhibitor. Neuropharmacology. 2014; 77:334-341.

43. Gonçalves D, Alves G, Fortuna A, Soares-da-Silva P, Falcao A. Pharmacokinetics of opicapone, a novel catecholomethyltransferase inhibitor, in the wistar rat. Parkinsonism Rel Disord. 2016; 22: e149ee192.

44. Loureiro AI, Fernandes-Lopes C, Soares-da-Silva P. Distribution, metabolism and elimination of opicapone in the rat and non-human primates. Parkinsonism \& Related Disorders. 2016; 22, Supplement 2: e184

45. Gonçalves D, Alves G, Fortuna A, et al. Pharmacokinetics of opicapone, a novel catecholO-methyltransferase inhibitor, in the Wistar rat. Parkinsonism \& Related Disorders. 2016; 22, Supplement 2: e184.

46. Gonçalves D, Alves G, Fortuna A, et al. An HPLC-DAD method for the simultaneous quantification of opicapone (BIA 9-1067) and its active metabolite in human plasma. Analyst. 2013; 138:2463-2469. 


\section{- Detection of active OPC metabolites in humans}

47. Rocha JF, Falcão A, Santos A, et al. Effect of opicapone and entacapone upon levodopa pharmacokinetics during three daily levodopa administrations. Eur J Clin Pharmacol. 2014; 70:1059-1071.

48. Rocha JF, Sicard É, Fauchoux N, et al. Effect of opicapone multiple-dose regimens on levodopa pharmacokinetics. Br J Clin Pharmacol. 2017; 83:540-553.

49. Rocha JF, Almeida L, Falcão A, et al. Opicapone: a short lived and very long acting novel catechol-O-methyltransferase inhibitor following multiple dose administration in healthy subjects. Br J Clin Pharmacol. 2013; 76:763-775.

50. Almeida L, Rocha JF, Falcão A, et al. Pharmacokinetics, pharmacodynamics and tolerability of opicapone, a novel catechol-O-methyltransferase inhibitor, in healthy subjects: prediction of slow enzyme-inhibitor complex dissociation of a short-living and very long-acting inhibitor. Clin Pharmacokinet. 2013; 52:139-151.

51. Bonifácio,M.J., Sousa, F, Soares-da-Silva, P. Potential drug toxicity of nitrocatechol COMT inhibitors in HepaRG. Toxicol. Lett. 2015; 238S:S319.

- An study of drug toxicity of COMT inhibitors in HepaRG

52. Lopes N, Ferreira J, Lees A, et al. Hepatic safety of opicapone in Parkinson's disease patients. Move Disord. 2015;30 Suppl 1:263.

53. Lees A, Ferreira JJ, Rocha JF, et al. Safety Profile of Opicapone in the Management of Parkinson's Disease. J Parkinsons Dis. 2019; 9:733-740.

54. Costa R, Oliveira C, Pinto R, et al. One-year open-label efficacy and safety of opicapone in Parkinson's disease BIPARK-II study. Move Disord. 2014; 29 Suppl 1:630.

55. Pinto R, Vaz-da-Silva M, Lopes N, et al. Cardiac safety of opicapone in patients with Parkinson's disease: analysis of the centralized phase III ECG dataset. Mov Disord. 2015;30 Suppl 1:294.

56. Pinto R, l'Hostis P, Patat A, et al. Evaluation of opicapone on cardiac repolarization in a thorough QT/QTc study. Clin Pharmacol Drug Dev. 2015; 4: 454-62. 
57. Castro Caldas A, Teodoro T, Ferreira JJ. The launch of opicapone for Parkinson's disease: negatives versus positives. Expert Opin Drug Saf. 2018; 17:331-337.

58. Ferreira JJ, Lees A, Rocha JF, et al. Bi-Park 1 investigators. Opicapone as an adjunct to levodopa in patients with Parkinson's disease and end-of-dose motor fluctuations: a randomised, double-blind, controlled trial. Lancet Neurol. 2016; 15:154-165.

59. Lees AJ, Ferreira J, Rascol O, et al. BIPARK-2 Study Investigators. Opicapone as Adjunct to Levodopa Therapy in Patients with Parkinson Disease and Motor Fluctuations: A Randomized Clinical Trial. JAMA Neurol. 2017;74:197-206.

60. Lopes N, Ferreira J, Lees A, et al. Exploratory efficacy of opicapone in fluctuating Parkinson's disease patients at different stages of symptom progression. Mov Disord. 2016; 31(suppl 2):S642.

61. Lees A, Ferreira J, Lopes N, et al. Efficacy and safety of opicapone in patients over 70 years with Parkinson's disease and motor fluctuations. Mov Disord. 2015;30 (suppl 1):S99.

62. Ferreira J, Lees A, Rocha J, et al. Efficacy and safety of opicapone in patients with Parkinson's disease and motor fluctuations: 1-year follow-up (bipark I). J Neurol Sci. 2015; 357: e255-e294.

63. Ferreira JJ, Rocha JF, Falcão A, et al. Effect of opicapone multiple-dose regimens on levodopa pharmacokinetics, motor response, and erythrocyte-COMT activity in Parkinson's patients co-administered with levodopa/dopa-decarboxylase inhibitor. J Neurol Sci. 2013; 333, Suppl 1, e116.

64. Lees A, Ferreira JJ, Costa R, et al. Efficacy and safety of opicapone, a new COMTinhibitor, for the treatment of motor fluctuations in Parkinson's Disease patients: BIPARKII studyA. J Neurol Sci. 2013; 333, Suppl 1, e116.

65. Rocha JF, Falcão AR, Pinto R, et al. Effect of opicapone and entacapone on levodopa pharmacokinetics when administered with immediate release 100/25 mg levodopa/carbidopa in healthy subjects. J Neurol Sci. 2013; 333, Suppl 1, e115. 
66. Bonifacio MJ, Torrao LS, Loureiro AI, et al. Opicapone: Characterization of a novel peripheral long-acting catechol-o-methyltransferase inhibidor. Parkinsonism Rel Disord. 2012; 18S2: S81-S159.

67. Ferreira, JJ, Lees, A., Rocha, J, et al. Efficacy and safety of opicapone in patients with Parkinson's disease and motor fluctuations: 1- year follow-up (Bipark I). J. Neurol. Sci.2015: 357, e285

68. Lees AJ, Ferreira J, Rascol O, et al. Opicapone for the management of end-of-dose motor fluctuations in patients with Parkinson's disease treated with L-DOPA. Expert Rev Neurother. 2017; 17:649-659.

69. Fabbri M, Ferreira JJ, Lees A, et al. Opicapone for the treatment of Parkinson's disease: A review of a new licensed medicine. Mov Disord. 2018; 33:1528-1539.

70. Ferreira J, Lees A, Tolosa E, et al. Switching double-blind opicapone, entacapone or placebo to open-label opicapone: efficacy results of the 1-year extension of study BIPARK I. Mov Disord. 2016; 31 (suppl 2):S633.

71. Ferreira J, Lees A, Rascol O, et al. Activities of daily living and motor scores of the UPDRS in fluctuating Parkinson's disease treated with opicapone. Mov Disord. 2016;31(suppl 2):S643.

72. Costa R, Oliveira C, Pinto R, et al. One-year open-label efficacy and safety of opicapone in Parkinson's disease BIPARK-II study. Mov Disord. 2014;29(suppl 1):S233

73. Ferreira J, Lees A, Lopes N, et al. Pooled efficacy of opicapone as adjunctive therapy to levodopa in patients with Parkinson's disease and motor fluctuations. Parkinsonism Rel Disord. 2016; 22: e87ee141.

74. Lees A, Ferreira J, Gama H, et al. Pooled safety and tolerability of opicapone in the treatment of Parkinson's disease and motor fluctuations. Parkinsonism Rel Disord. 2016; 22: e87ee141.

75. Ferreira JJ, Lees A, Rocha JF, et al. Long-term efficacy of opicapone in fluctuating Parkinson's disease patients: a pooled analysis of data from two phase 3 clinical trials and their open-label extensions. Eur J Neurol. 2019;26:953-960. 
76. GlobalData (2014). Parkinson's Disease - Current and Future Players, March 2014, GDHC1033FPR

77. GlobalData (2014). Opicapone (Parkinson's Disease) - Forecast and Market Analysis to 2022, March 2014, GDHC402DFR

78. Ferreira JJ, Lees AJ, Poewe W, et al. Effectiveness of opicapone and switching from entacapone in fluctuating Parkinson disease. Neurology. 2018; 90:e1849-e1857.

79. Bet L, Bareggi SR, PaceiF, et al. Bimodal administration of entacapone in Parkinson's disease patients improves motor control. Eur J Neurol. 2008; 15:268-273.

80. Kaakkola S. Problems with the present inhibitors and a relevance of new and improved COMT inhibitors in Parkinson's disease. Int Rev Neurobiol. 2010;95:207-25.

81. Gordin A, Kaakkola S, Teräväinen H. Clinical advantages of COMT inhibition with entacapone - a review. J Neural Transm(Vienna). 2004;111:1343-1363.

82. Ferreira JJ, Katzenschlager R, Bloem BR, et al. Summary of the recommendations of the EFNS/MDS-ES review on therapeutic management of Parkinson's disease. Eur J Neurol. $2013 ; 20: 5-15$.

83. The Parkinson Study Group QE3 Investigators. A randomized clinical trial of high dosage Coenzyme Q10 in early Parkinson disease: no evidence of benefit. JAMA Neurol. 2014; 71:543-552.

84. Jankovic J, Goodman I, Safirstein B, et al. Safety and tolerability of Multiple ascending doses of PRX002/RG7935, an anti- $\alpha$-Synuclein monoclonal antibody, in patients with parkinson disease: a randomized clinical trial. JAMA Neurol. 2018; 75:1206-1214.

85. Schenk DB, Koller M, Ness DK, et al. First-in-human assessment of PRX002, an anti- $\alpha$ synuclein monoclonal antibody, in healthy volunteers. Mov Disord. 2017; 32:211-218.

86. Marks WJ Jr, Baumann TL, Bartus RT. Long-Term Safety of Patients with Parkinson's Disease Receiving rAAV2-Neurturin (CERE-120) Gene Transfer. Hum Gene Ther. 2016; 27:522-527. 
87. Bartus RT, Baumann TL, Siffert J, et al. Safety/feasibility of targeting the substantia nigra with AAV2-neurturin in Parkinson patients. Neurology. 2013; 80:1698-701.

88. Blits B, Petry H. Perspective on the Road toward Gene Therapy for Parkinson's disease. Front Neuroanat. 2017; 10:128.

89. Whone A, Luz M, Boca M, et al. Randomized trial of intermittent intraputamenal glial cell line-derived neurotrophic factor in Parkinson's disease. Brain. 2019;142:512-525.

90. Whone AL, Boca M, Luz M et al. Extended Treatment with Glial Cell Line-Derived Neurotrophic Factor in Parkinson's disease. J Parkinsons Dis. 2019;9:301-313.

Figure 1. The co-administration of L-DOPA / DDI with OPC (peripheral COMT inhibitor), allows a more continuous supply of L-DOPA in the brain. Therefore, OPC improves the symptomatology of PD by decreasing fluctuations in plasma levels of L-DOPA and favoring a constant stimulation of the central dopaminergic receptor by increasing brain dopamine levels. 